\title{
Research of efficiency of application of external physical fields for sedimentation of liquid- droplets aerosols of different concentration
}

\author{
Maria Yu. Stepkina ${ }^{1, *}$, Olga B.Kudryashova ${ }^{1}$, and Natalia V. Korovina ${ }^{1}$ \\ ${ }^{1}$ Institute for Problems of Chemical and Energetic Technologies of the SB RAS, 659322 Biisk, Russia
}

\begin{abstract}
A theoretical estimation of extent of influence of physical and chemical properties and concentration of the weighed particles on evolution of parameters of a liquid-droplets aerosol is carried out in the work. A series of experiments on a research of parameters of aerosol media with a various structure and concentration characteristics of a dispersive phase under external (ultrasonic and electrostatic) fields were made.
\end{abstract}

\section{Introduction}

In the industry, the modern equipment, technology and everyday life it is necessary to consider the processes and the phenomena proceeding with the participation of aerosol systems and powdery materials. The research of aerosols and aero-disperse systems is extremely important for the organization of environmental protection and health safety. Formation of an aerosol happens with mechanical fragmentation and dispersion of solids or liquids, crushing, friction, explosions, burning, dispersion by sprayers.

Now the problem of aerosols creation for needs of fire extinguishing, disinfection, deactivation, the creation of barriers on the way of distribution of toxic gases, non-lethal weapon, inhalation in medicine, etc. is relevant.

No matter how the liquid-droplets aerosol has been received, the cloud evolves further: liquid droplets evaporate or increase in the course of condensation of vapors, particles stick together or split up, fall down. Acceleration of process of sedimentation of the considered aerosol media is increased under external physical fields [1,2].

\section{Theoretical part}

An evaporation of the condensed phase of a liquid-droplets aerosol makes the important contribution to the process of the transformation of the size range of aerosol-cloud particles. Most strongly evaporation processes influence on particles less than 10 microns in size, therefore, these processes need to be taking into account by consideration of ensemble of such particles. Such influence is caused by the active heat exchange with the environment and energy ablation from the large surface of droplets.

\footnotetext{
${ }^{*}$ Corresponding author: bvborisov@tpu.ru
} 
For calculation of time of evaporation of $t$ on the basis of Maxwell's equation the following formula is used [3]:

$$
t=\frac{r^{2}}{\frac{6 D_{f} M}{\rho_{K} R T_{\infty}} e^{-\frac{M H}{R}\left(\frac{1}{T_{K}}-\frac{1}{293}\right)} 2338.8\left(e^{\frac{2 \sigma M}{\rho_{K} R T_{K} \cdot r}}-v\right)},
$$

where $\rho_{\kappa}$ is the droplets density; $D_{f}$ is diffusion coefficient; $M$ is molecular weight of liquid; $r$ is the particle radius; $R$ is universal gas constant, $\sigma$ is coefficient of a surface tension of substance of a droplet; $T_{\kappa}$ is the droplet temperature; $\mathrm{v}$ is humidity; $T_{\infty}$ is the temperature of the air.

An example of the calculation is presented for the dependence of evaporation time of aerosol particles with a diameter 1-15 micrometers for substances - water, acetone, ethyl alcohol (Fig.1), hydrochloric acid and glycerin (Fig.2) at an ambient temperature of $20 \mathrm{o} \mathrm{C}$ and humidity of $70 \%$.

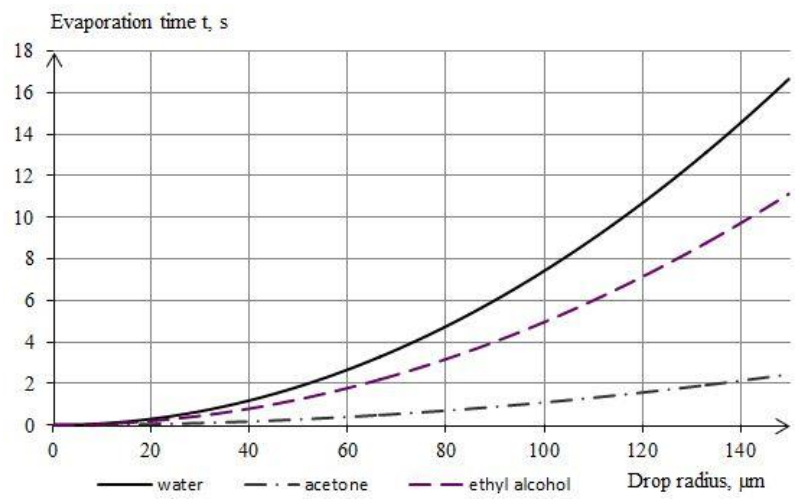

Fig. 1. Time of evaporation of aerosol particles of water, acetone, ethyl alcohol at ambient temperature $20^{\circ} \mathrm{C}$.

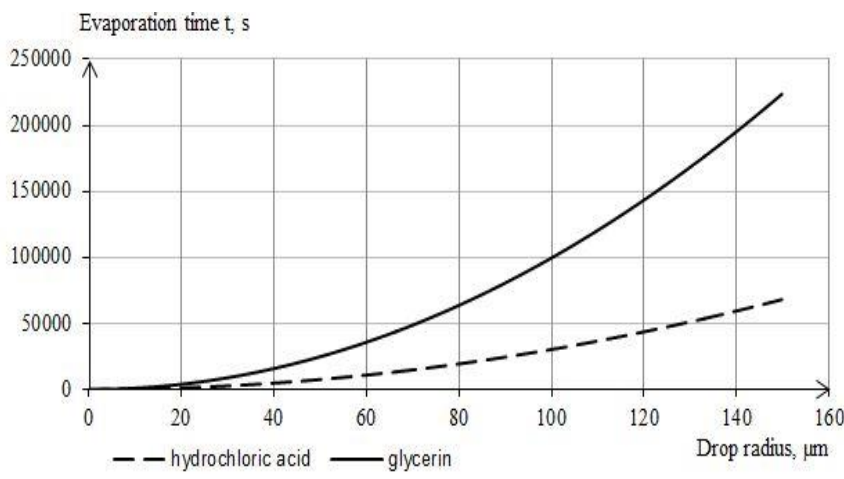

Fig. 2. Time of evaporation of aerosol particles of hydrochloric acid and glycerin at ambient temperature $20^{\circ} \mathrm{C}$.

Transformation of distribution of particles by the sizes is based on the balance equation (an integrated variant of the equation of Smolukhovsky), as following [6]:

$$
\frac{\partial f(D, t)}{\partial t}=I_{1}+I_{2}+I_{3}
$$


where $I_{1}$ is a decrease in mass of droplets with a diameter of $D$ in a unit of time, in unit of volume due to collision of a droplet of diameter $D$ with any droplet of diameter $D^{\prime} ; I_{2}$ describes formation of particles of diameter of $D$ due to collision of droplets with diameters $D^{\prime}$ and $D-D^{\prime} ; I_{3}$ is the reduction of mass of droplets due to their evaporation.

The term of $I_{1}$ is calculated by a formula:

$$
I_{1}=-f(D, t) \int_{0}^{\infty} K\left(D, D^{\prime}\right) f\left(D^{\prime}, t\right) d D^{\prime},
$$

where $K\left(D, D^{\prime}\right)=b_{k}\left(D^{2}+D^{\prime 2}\right)$ is the probability of collision of drops with diameters of $D$ and $D^{\prime}$ in the unit of time.

For the calculation of $I_{2}$ a formula is used:

$$
I_{2}=\frac{1}{2} \int_{0}^{D} K\left(D-D^{\prime}, D^{\prime}\right) f\left(D^{\prime}, t\right) f\left(D-D^{\prime}, t\right) d D^{\prime} .
$$

The term is defined by Maxwell (1) equation with Thomson formula differentiated on the mass of particles:

$$
I_{3}=\frac{\partial}{\partial D}\left[4 \pi D_{f} M p_{p l}\left(\exp \left(\frac{4 \sigma M}{\rho_{l} R T D}\right)-1\right) \frac{f(D)}{R T D \rho_{l}}\right]
$$

It is necessary to consider initial conditions for the equation (1) at $t=t_{0} f\left(D, t_{0}\right)=f_{0}(D)-$ initial distribution particles by the sizes.

In the course of sedimentation of an aerosol the particle, which weight exceeds critical value instantly drop out in a deposit and don't take part in coagulation. However, to track dynamics of the process of sedimentation it is necessary to consider the dependence of the critical size on time, for this purpose it is necessary to write down the equation for sedimentation speed as particle derivative of height above the ground of $h$ on time:

$$
V_{o}=\frac{d h}{d t}=\frac{2}{9} \frac{D^{2} g\left(\rho_{k}-\rho_{0}\right)}{\mu_{g}}
$$

Then in the time of $t$ all the droplets with the diameter more than $D_{\text {cr }}$ will drop out in a deposit. The value of $D_{\text {cr }}$ can be expressed from the equation (6) as:

$$
D_{c r}=\sqrt{\frac{9 \mu_{g} h}{2 g\left(\rho_{\kappa}-\rho_{0}\right) t}},
$$

Then the equation (3) taking into account of $D_{\mathrm{cr}}$ will take a form:

$$
I_{1}=-f(D, t) \int_{0}^{D_{c r}(t)} K\left(D, D^{\prime}\right) f\left(D^{\prime}, t\right) d D^{\prime}
$$

Thus, considering sedimentation of large particles, the range of the particle sizes on each time point will be cut off on the right and gradually this border is displaced towards smaller particles [4-5]. 


\section{Experimental part}

Experimental study of the influence of external fields on the evolution of parameters of a liquid-droplets aerosol was carried out in the measuring volume of $1 \mathrm{~m} 3$. For the creation of a liquid-droplets aerosol, the airbrush "CRATON R 200 LVLP-02S" was used. The research of evolution of parameters of a liquid-droplets aerosol in the experimental camera was measured by "LID-2M" system because this system is intended for measurement of dispersion and concentration of aerosol particles from the moment of aerosol generation till the total disappearance of it. The principle of action of the measurement system is based on the modified method of low-angle scattering representing a contactless method of determination of the dispersing characteristics of the aerosol based on a classical method of low-angle scattering [7].

For the creation of external fields in experimental researches, the electro precipitator and the ultrasonic radiator are used. Technical characteristics of the ultrasonic device are following: diameter of the radiator $-320 \mathrm{~mm}$, the level of sound pressure - not less than 140 $\mathrm{dB}$, the frequency of fluctuations $-28 \mathrm{kHz}$. Design data of the electric precipitator used in an experiment are the following: the area of sedimentation is $1000 \mathrm{~mm} 2$; length of a discharge element, length of an active zone $-0.085 \mathrm{~m}$; distance between discharge electrodes -0.01 $\mathrm{m}$; distance between the planes of discharge and precipitation electrodes $-0.005 \mathrm{~m}$; radius of a discharge electrode $-0.004 \mathrm{~m}$; average tension $-8000 \mathrm{~V}$ [8].

The mass of the sprayed water solution of $20 \%$ of glycerin in experiments was $9.5 \mathrm{~g}$; the time of the spraying is 35 seconds; initial humidity is $40 \%$ and the temperature is $20 \mathrm{o} \mathrm{C}$. The time of registration of process is more than one hour. After the spraying we start the registration of the process of sedimentation within 30 minutes, and then we turn on the precipitator or the ultrasonic radiator for 1 hour in experiments with external fields.

The values of a decrease of relative concentration of $\mathrm{Cm}$ for glycerin solution are received as a result of an experiment using the software of the "LID-2M" laser system. The first series of experiments were carried out without any influences under normal conditions (Fig.3). Also calculations of time of sedimentation taking into account evaporation, coagulation and sedimentations have been carried out (the equation (2) taking into account or without the member of I3). The following series of experiments were carried out in the presence of acoustic and electrostatic field (Fig.4) in identical conditions of an experiment.

The calculated curve taking into account the only sedimentation of particles predicts sedimentation of an aerosol already to 1500 second. Evaporation of droplets reduces their size and they remain in the air longer, without sedimentation. Both calculation and an experiment show this fact (Fig.3).

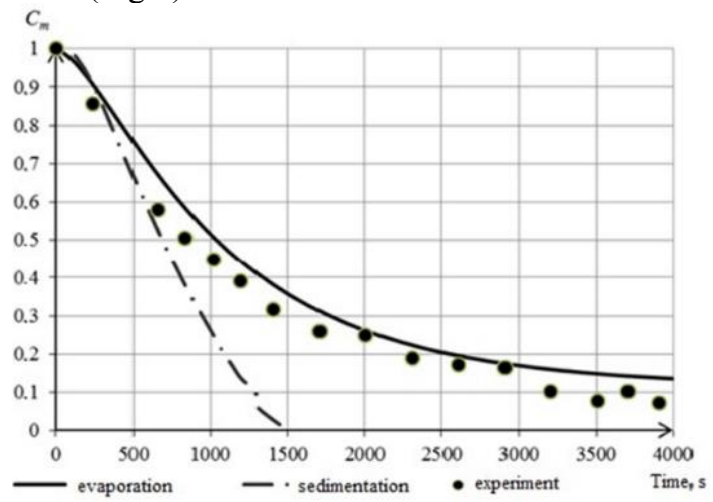

Fig. 3. Dependence of relative concentration of aerosol particles of $20 \%$ solution of glycerin on time at a humidity of $40 \%$ and temperature of the disperse media of $20^{\circ} \mathrm{C}$. 


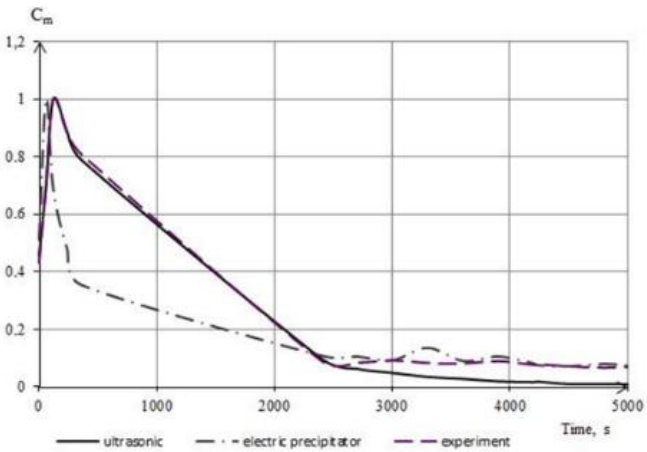

Fig. 4. Experimental dependences of the relative concentration of aerosol particles of $20 \%$ water solution of glycerin on time without influences and at under external fields with humidity of $40 \%$ and temperature of the disperse media of $20^{\circ} \mathrm{C}$.

Under the external field of ultrasound (US) the experimental curve of concentration tends to zero after $4000 \mathrm{sec}$., that allows confirming a good result of sedimentation of an aerosol of the small sizes of particles under the field. Influence of electrostatic field allows to remove quickly from the air the large particles, but smaller ones continue to remain in the air for a long time.

\section{Conclusion}

The theoretical estimation of the extent of influence of physical and chemical properties of a dispersed phase on the evolution of parameters of liquid-droplets aerosols allowing to predict the evaporation time of an aerosol of different substances is carried out. It has been as a result established (Fig.1-2) that time of evaporation of large particles (140 micrometers) of glycerin will be more than 55 hours. Among a number of model liquid-droplets particles, the acetone is the most easily evaporated substance. The experimental trends of a decrease of relative concentration received by means of laser measuring system have allowed revealing the most effective mechanism of sedimentation on the example of an aerosol of $20 \%$ of a water solution of glycerin: it turned out the ultrasonic sedimentation.

This study was supported by the Russian Science Foundation (grant No. 17-79-10209).

The work was conducted on equipment of the Biysk Regional Center of Collective Use of the SB RAS (IPCET SB RAS, Biysk).

\section{References}

1. V.I. Roldugin, Fizikokhimiya poverkhnosti (Intellekt, Moscow, 2008) [In Russian]

2. V.A. Arkhipov, A.S. Usanina, The movement of aerosol particles in a stream (Publishing house of the Tomsk state university, Tomsk, 2013)

3. M.Y. Stepkina, O.B. Kudryashova, B.V. Borisov, MATEC Web Conf. 23 (2015)

4. A.A. Antonnikova, N.V. Korovina, O.B. Kudryashova, I.M. Vasenin, Polzunovsky vestnik 1 (2013) [In Russian]

5. O.B. Kudryashova, A.A. Antonnikova, Tomsk Polytechnic University News 322 (2013)

6. A.A. Antonnikova, N.V. Korovina, O. B. Kudryashova, OJA E 3, 3 (2013)

7. O. Kudryashova, Photodetectors (InTech, Croatia, 2012)

8. M.Y. Stepkina, O.B. Kudryashova, A.A. Antonnikova, Arch Acoust. 43 (2018) 